\title{
Polish Notation
}

\author{
Taneli Huuskonen ${ }^{1}$ \\ Department of Mathematics and Statistics \\ University of Helsinki \\ Finland
}

Summary. This article is the first in a series formalizing some results in my joint work with Prof. Joanna Golińska-Pilarek ([12] and [13]) concerning a logic proposed by Prof. Andrzej Grzegorczyk (14]).

We present some mathematical folklore about representing formulas in "Polish notation", that is, with operators of fixed arity prepended to their arguments. This notation, which was published by Jan Łukasiewicz in 15, eliminates the need for parentheses and is generally well suited for rigorous reasoning about syntactic properties of formulas.

MSC: 68R15 03B35

Keywords: Polish notation; syntax; well-formed formula

MML identifier: POLNOT_1, version: 8.1.04 5.32.1240

The notation and terminology used in this paper have been introduced in the following articles: [5], [1, 4], [11, 4], 8], 3], 9], [16], [19], [17, [18, and [10].

\section{Preliminaries}

From now on $k, m, n$ denote natural numbers, $a, b, c, c_{1}, c_{2}$ denote objects, $x, y, z, X, Y, Z$ denote sets, $D$ denotes a non empty set, $p, q, r, s, t, u, v$ denote finite sequences, $P, Q, R, P_{1}, P_{2}, Q_{1}, Q_{2}, R_{1}, R_{2}$ denote finite sequencemembered sets, and $S, T$ denote non empty, finite sequence-membered sets.

Let $D$ be a non empty set and $P, Q$ be subsets of $D^{*}$. The functor $\frown(D, P, Q)$ yielding a subset of $D^{*}$ is defined by the term

\footnotetext{
${ }^{1}$ Work supported by Polish National Science Center (NCN) grant "Logic of language experience" nr 2011/03/B/HS1/04580. 
(Def. 1) $\quad\left\{p^{\frown} q\right.$, where $p$ is a finite sequence of elements of $D, q$ is a finite sequence of elements of $D: p \in P$ and $q \in Q\}$.

Let us consider $P$ and $Q$. The functor $P \frown Q$ yielding a finite sequencemembered set is defined by

(Def. 2) for every $a, a \in i$ iff there exists $p$ and there exists $q$ such that $a=p^{\frown} q$ and $p \in P$ and $q \in Q$.

Let $\beta$ be an empty set. One can check that $\beta \frown P$ is empty and $P \frown \beta$ is empty.

Let us consider $S$ and $T$. One can check that $S^{\frown} T$ is non empty.

Now we state the propositions:

(1) If $p^{\frown} q=r^{\frown} s$, then there exists $t$ such that $p^{\frown} t=r$ or $p=r^{\frown} t$.

(2) $(P \frown Q) \frown R=P \frown(Q \frown R)$.

Proof: For every $a, a \in\left(P^{\frown} Q\right)^{\frown} R$ iff $a \in P^{\frown}\left(Q^{\frown} R\right)$ by [4, (32)].

Note that $\{\emptyset\}$ is non empty and finite sequence-membered.

(i) $P \frown\{\emptyset\}=P$, and

(ii) $\{\emptyset\} \frown P=P$.

Proof: For every $a, a \in P \frown\{\emptyset\}$ iff $a \in P$ by [4, (34)]. For every $a$, $a \in\{\emptyset\}^{\frown} P$ iff $a \in P$ by [4, (34)].

Let us consider $P$. The functor $P \frown \frown$ yielding a function is defined by

(Def. 3) $\operatorname{dom} i t=\mathbb{N}$ and $i t(0)=\{\emptyset\}$ and for every $n$, there exists $Q$ such that $Q=i t(n)$ and $i t(n+1)=Q^{\frown} P$.

Let us consider $n$. The functor $P \frown n$ yielding a finite sequence-membered set is defined by the term

(Def. 4) $(P \frown)(n)$.

Now we state the proposition:

(4) $\emptyset \in P \frown 0$.

Let us consider $P$. Let $n$ be a zero natural number. Note that $P \frown n$ is non empty.

Let $\beta$ be an empty set and $n$ be a non zero natural number. One can verify that $\beta \frown n$ is empty.

Let us consider $P$. The functor $P^{*}$ yielding a non empty, finite sequencemembered set is defined by the term

(Def. 5) Uthe set of all $P^{\frown} n$ where $n$ is a natural number.

(5) $a \in P^{*}$ if and only if there exists $n$ such that $a \in P^{\frown} n$.

Let us consider $P$.

(6) (i) $P \frown 0=\{\emptyset\}$, and

(ii) for every $n, P \frown(n+1)=(P \frown n) \frown P$. 
(7) $P \frown 1=P$. The theorem is a consequence of (6) and (3).

(8) $P \frown n \subseteq P^{*}$.

(9) (i) $\emptyset \in P^{*}$, and

(ii) $P \subseteq P^{*}$.

The theorem is a consequence of (4), (5), and (7).

(10) $P \frown(m+n)=(P \frown m) \frown(P \frown n)$.

Proof: Define $\mathcal{X}$ [natural number] $\equiv P \frown\left(m+\$_{1}\right)=(P \frown m) \frown\left(P \frown \$_{1}\right)$. $\mathcal{X}[0]$. For every $k$ such that $\mathcal{X}[k]$ holds $\mathcal{X}[k+1]$. For every $k, \mathcal{X}[k]$ from [2, Sch. 2].

(11) If $p \in P \frown m$ and $q \in P \frown n$, then $p^{\frown} q \in P \frown(m+n)$. The theorem is a consequence of (10).

(12) If $p, q \in P^{*}$, then $p^{\frown} q \in P^{*}$. The theorem is a consequence of (5) and (11).

(13) If $P \subseteq R^{*}$ and $Q \subseteq R^{*}$, then $P \frown Q \subseteq R^{*}$. The theorem is a consequence of $(12)$.

(14) If $Q \subseteq P^{*}$, then $Q \frown n \subseteq P^{*}$.

Proof: Define $\mathcal{X}$ [natural number $] \equiv Q \frown \$_{1} \subseteq P^{*} . \mathcal{X}[0]$. For every $k$ such that $\mathcal{X}[k]$ holds $\mathcal{X}[k+1]$. For every $k, \mathcal{X}[k]$ from [2, Sch. 2].

(15) If $Q \subseteq P^{*}$, then $Q^{*} \subseteq P^{*}$. The theorem is a consequence of (5) and (14).

(16) If $P_{1} \subseteq P_{2}$ and $Q_{1} \subseteq Q_{2}$, then $P_{1} \frown Q_{1} \subseteq P_{2} \frown Q_{2}$.

(17) If $P \subseteq Q$, then for every $n, P \frown n \subseteq Q \frown n$.

Proof: Define $\mathcal{S}$ [natural number] $\equiv P \frown \$_{1} \subseteq Q \frown \$_{1} . P \frown 0=\{\emptyset\}$. For every $n$ such that $\mathcal{S}[n]$ holds $\mathcal{S}[n+1]$. For every $n, \mathcal{S}[n]$ from [2, Sch. 2].

Let us consider $S$ and $n$. Let us observe that $S \frown n$ is non empty and finite sequence-membered.

\section{The Language}

In the sequel $\alpha$ denotes a function from $P$ into $\mathbb{N}$ and $U, V, W$ denote subsets of $P^{*}$.

Let us consider $P, \alpha$, and $U$. The $\operatorname{Polish}$-expression $\operatorname{layer}(P, \alpha, U)$ yielding a subset of $P^{*}$ is defined by

(Def. 6) for every $a, a \in$ it iff $a \in P^{*}$ and there exists $p$ and there exists $q$ and there exists $n$ such that $a=p \frown q$ and $p \in P$ and $n=\alpha(p)$ and $q \in U^{\frown} n$.

Now we state the proposition:

(18) Suppose $p \in P$ and $n=\alpha(p)$ and $q \in U^{\frown} n$. Then $p^{\frown} q \in$ the Polish-expression layer $(P, \alpha, U)$. The theorem is a consequence of (14), (9), and (12). 
Let us consider $P$ and $\alpha$. The Polish atoms $(P, \alpha)$ yielding a subset of $P^{*}$ is defined by

(Def. 7) for every $a, a \in$ it iff $a \in P$ and $\alpha(a)=0$.

The Polish operations $(P, \alpha)$ yielding a subset of $P$ is defined by the term

(Def. 8) $\left\{t\right.$, where $t$ is an element of $P^{*}: t \in P$ and $\left.\alpha(t) \neq 0\right\}$.

Now we state the propositions:

(19) The Polish atoms $(P, \alpha) \subseteq$ the Polish-expression layer $(P, \alpha, U)$. The theorem is a consequence of (4) and (18).

(20) Suppose $U \subseteq V$. Then the Polish-expression layer $(P, \alpha, U) \subseteq$ the Polishexpression layer $(P, \alpha, V)$. The theorem is a consequence of (17).

(21) Suppose $u \in$ the Polish-expression layer $(P, \alpha, U)$. Then there exists $p$ and there exists $q$ such that $p \in P$ and $u=p^{\frown} q$.

Let us consider $P$ and $\alpha$. The Polish-expression hierarchy $(P, \alpha)$ yielding a function is defined by

(Def. 9) $\operatorname{dom} i t=\mathbb{N}$ and $i t(0)=$ the $\operatorname{Polish} \operatorname{atoms}(P, \alpha)$ and for every $n$, there exists $U$ such that $U=i t(n)$ and $i t(n+1)=$ the Polish-expression layer $(P, \alpha, U)$.

Let us consider $n$. The Polish-expression hierarchy $(P, \alpha, n)$ yielding a subset of $P^{*}$ is defined by the term

(Def. 10) (the Polish-expression hierarchy $(P, \alpha))(n)$.

Now we state the proposition:

(22) The Polish-expression hierarchy $(P, \alpha, 0)=$ the $\operatorname{Polish}$ atoms $(P, \alpha)$.

Let us consider $P, \alpha$, and $n$. Now we state the propositions:

(23) The Polish-expression hierarchy $(P, \alpha, n+1)=$ the Polish-expression layer $(P, \alpha$, the Polish-expression hierarchy $(P, \alpha, n))$.

(24) The Polish-expression hierarchy $(P, \alpha, n) \subseteq$ the Polish-expression hierarchy $(P, \alpha, n+1)$.

Proof: Define $\mathcal{S}$ [natural number $] \equiv$ the Polish-expression hierarchy $(P$, $\left.\alpha, \$_{1}\right) \subseteq$ the Polish-expression hierarchy $\left(P, \alpha, \$_{1}+1\right)$. $\mathcal{S}[0]$. For every $k$ such that $\mathcal{S}[k]$ holds $\mathcal{S}[k+1]$. For every $k, \mathcal{S}[k]$ from [2, Sch. 2].

Now we state the proposition:

(25) The Polish-expression hierarchy $(P, \alpha, n) \subseteq$ the Polish-expression hierarchy $(P, \alpha, n+m)$.

Proof: Define $\mathcal{S}$ [natural number] $\equiv$ the Polish-expression hierarchy $(P$, $\alpha, n) \subseteq$ the Polish-expression hierarchy $\left(P, \alpha, n+\$_{1}\right)$. For every $k$ such that $\mathcal{S}[k]$ holds $\mathcal{S}[k+1]$. For every $k, \mathcal{S}[k]$ from [2, Sch. 2]. 
Let us consider $P$ and $\alpha$. The Polish-expression $\operatorname{set}(P, \alpha)$ yielding a subset of $P^{*}$ is defined by the term

(Def. 11) Uthe set of all the Polish-expression hierarchy $(P, \alpha, n)$ where $n$ is a natural number.

Now we state the propositions:

(26) The Polish-expression hierarchy $(P, \alpha, n) \subseteq$ the Polish-expression set $(P$, $\alpha)$.

(27) Suppose $q \in(\text { the Polish-expression } \operatorname{set}(P, \alpha))^{\frown} n$. Then there exists $m$ such that $q \in(\text { the Polish-expression hierarchy }(P, \alpha, m))^{\frown} n$.

Proof: Define $\mathcal{S}$ [natural number] $\equiv$ for every $q$ such that $q \in$ (the Polishexpression $\operatorname{set}(P, \alpha) \frown \$_{1}$ there exists $m$ such that $q \in$ (the Polish-expression hierarchy $(P, \alpha, m)) \frown \$_{1} \cdot \mathcal{S}[0]$. For every $k$ such that $\mathcal{S}[k]$ holds $\mathcal{S}[k+1]$. For every $k, \mathcal{S}[k]$ from [2, Sch. 2].

(28) Suppose $a \in$ the Polish-expression $\operatorname{set}(P, \alpha)$. Then there exists $n$ such that $a \in$ the Polish-expression hierarchy $(P, \alpha, n+1)$. The theorem is a consequence of (24).

Let us consider $P$ and $\alpha$.

A Polish expression of $P$ and $\alpha$ is an element of the Polish-expression $\operatorname{set}(P$, $\alpha$ ). Let us consider $n$ and $t$. Assume $t \in P$. The $\operatorname{Polish} \operatorname{operation}(P, \alpha, n, t)$ yielding a function from (the Polish-expression $\operatorname{set}(P, \alpha))^{\frown} n$ into $P^{*}$ is defined by

(Def. 12) for every $q$ such that $q \in \operatorname{dom} i t$ holds $i t(q)=t \frown q$.

Let us consider $X$ and $Y$. Let $F$ be a partial function from $X$ to $2^{Y}$. One can check that $F$ is disjoint valued if and only if the condition (Def. 13) is satisfied.

(Def. 13) for every $a$ and $b$ such that $a, b \in \operatorname{dom} F$ and $a \neq b$ holds $F(a)$ misses $F(b)$.

Let $X$ be a set. One can check that there exists a finite sequence of elements of $2^{X}$ which is disjoint valued.

Now we state the proposition:

(29) Let us consider a set $X$, a disjoint valued finite sequence $B$ of elements of $2^{X}, a, b$, and $c$. If $a \in B(b)$ and $a \in B(c)$, then $b=c$ and $b \in \operatorname{dom} B$.

Let us consider $X$. Let $B$ be a disjoint valued finite sequence of elements of $2^{X}$. The arity from list $B$ yielding a function from $X$ into $\mathbb{N}$ is defined by

(Def. 14) for every $a$ such that $a \in X$ holds there exists $n$ such that $a \in B(n)$ and $a \in B(i t(a))$ or there exists no $n$ such that $a \in B(n)$ and $i t(a)=0$.

Now we state the propositions:

(30) Let us consider a disjoint valued finite sequence $B$ of elements of $2^{X}$, and $a$. Suppose $a \in X$. Then (the arity from list $B)(a) \neq 0$ if and only if 
there exists $n$ such that $a \in B(n)$. The theorem is a consequence of (29).

(31) Let us consider a disjoint valued finite sequence $B$ of elements of $2^{X}$, $a$, and $n$. Suppose $a \in B(n)$. Then (the arity from list $B)(a)=n$. The theorem is a consequence of (29).

(32) Suppose $r \in$ the Polish-expression $\operatorname{set}(P, \alpha)$. Then there exists $n$ and there exists $p$ and there exists $q$ such that $p \in P$ and $n=\alpha(p)$ and $q \in(\text { the Polish-expression } \operatorname{set}(P, \alpha))^{\frown} n$ and $r=p^{\frown} q$. The theorem is a consequence of (28), (23), (26), and (17).

Let us consider $P, \alpha$, and $Q$. We say that $Q$ is $\alpha$-closed if and only if

(Def. 15) for every $p, n$, and $q$ such that $p \in P$ and $n=\alpha(p)$ and $q \in Q \frown n$ holds $p^{\frown} q \in Q$.

Now we state the propositions:

(33) The Polish-expression $\operatorname{set}(P, \alpha)$ is $\alpha$-closed. The theorem is a consequence of (27), (18), (23), and (26).

(34) If $Q$ is $\alpha$-closed, then the Polish atoms $(P, \alpha) \subseteq Q$. The theorem is a consequence of (4).

(35) If $Q$ is $\alpha$-closed, then the Polish-expression hierarchy $(P, \alpha, n) \subseteq Q$.

Proof: Define $\mathcal{X}$ [natural number] $\equiv$ the Polish-expression hierarchy $(P$, $\left.\alpha, \$_{1}\right) \subseteq Q . \mathcal{X}[0]$. For every $k$ such that $\mathcal{X}[k]$ holds $\mathcal{X}[k+1]$. For every $k$, $\mathcal{X}[k]$ from [2, Sch. 2].

(36) The Polish atoms $(P, \alpha) \subseteq$ the Polish-expression $\operatorname{set}(P, \alpha)$. The theorem is a consequence of (33) and (34).

(37) If $Q$ is $\alpha$-closed, then the Polish-expression $\operatorname{set}(P, \alpha) \subseteq Q$. The theorem is a consequence of (28) and (35).

(38) Suppose $r \in$ the Polish-expression $\operatorname{set}(P, \alpha)$. Then there exists $n$ and there exists $t$ and there exists $q$ such that $t \in P$ and $n=\alpha(t)$ and $r=$ (the Polish operation $(P, \alpha, n, t))(q)$. The theorem is a consequence of (28), (23), (26), and (17).

(39) Suppose $p \in P$ and $n=\alpha(p)$ and $q \in($ the Polish-expression $\operatorname{set}(P, \alpha))$ $n$. Then (the Polish operation $(P, \alpha, n, p))(q) \in$ the Polish-expression $\operatorname{set}(P, \alpha)$. The theorem is a consequence of (33).

The scheme $A$ Ind deals with a finite sequence-membered set $\mathcal{P}$ and a function $\alpha$ from $\mathcal{P}$ into $\mathbb{N}$ and a unary predicate $\mathcal{X}$ and states that

(Sch. 1) For every $a$ such that $a \in$ the Polish-expression $\operatorname{set}(\mathcal{P}, \alpha)$ holds $\mathcal{X}[a]$ provided

- for every $p, q$, and $n$ such that $p \in \mathcal{P}$ and $n=\alpha(p)$ and $q \in\left(\right.$ the Polish-expression $\operatorname{set}(\mathcal{P}, \alpha) \frown^{\frown} n$ holds $\mathcal{X}\left[p^{\frown} q\right]$. 


\section{PARSING}

In the sequel $k, l, m, n, i, j$ denote natural numbers, $a, b, c, c_{1}, c_{2}$ denote objects, $x, y, z, X, Y, Z$ denote sets, $D, D_{1}, D_{2}$ denote non empty sets, $p, q, r$, $s, t, u, v$ denote finite sequences, and $P, Q, R$ denote finite sequence-membered sets.

Let us consider $P$. We say that $P$ is antichain-like if and only if

(Def. 16) for every $p$ and $q$ such that $p, p^{\frown} q \in P$ holds $q=\emptyset$.

Now we state the propositions:

(40) $P$ is antichain-like if and only if for every $p$ and $q$ such that $p, p^{\frown} q \in P$ holds $p=p^{\frown} q$.

Proof: If $P$ is antichain-like, then for every $p$ and $q$ such that $p, p^{\frown} q \in P$ holds $p=p^{\frown} q$ by [4, (34)].

(41) If $P \subseteq Q$ and $Q$ is antichain-like, then $P$ is antichain-like.

Note that every finite sequence-membered set which is trivial is also antichainlike.

Now we state the proposition:

(42) If $P=\{a\}$, then $P$ is antichain-like.

Note that there exists a non empty, finite sequence-membered set which is antichain-like and every finite sequence-membered set which is empty is also antichain-like.

An antichain is an antichain-like, finite sequence-membered set. In the sequel $B, C$ denote antichains.

Let us consider $B$. One can verify that every subset of $B$ is antichain-like and finite sequence-membered.

A Polish-language is a non empty antichain. From now on $S, T$ denote Polish-languages.

Let $D$ be a non empty set and $\psi$ be a subset of $D^{*}$. Note that $\psi$ is antichainlike if and only if the condition (Def. 17) is satisfied.

(Def. 17) for every finite sequence $g$ of elements of $D$ and for every finite sequence $h$ of elements of $D$ such that $g, g^{\frown} h \in \psi$ holds $h=\varepsilon_{D}$.

Now we state the proposition:

(43) If $p^{\frown} q=r^{\frown} s$ and $p, r \in B$, then $p=r$ and $q=s$. The theorem is a consequence of (1) and (40).

Let us consider $B$ and $C$. Note that $B \frown C$ is antichain-like.

Now we state the propositions:

(44) If for every $p$ and $q$ such that $p, q \in P$ holds $\operatorname{dom} p=\operatorname{dom} q$, then $P$ is antichain-like. 
Proof: For every $p$ and $q$ such that $p, p^{\frown} q \in P$ holds $p=p^{\frown} q$ by 4 , (21)].

(45) If for every $p$ such that $p \in P$ holds $\operatorname{dom} p=a$, then $P$ is antichain-like. The theorem is a consequence of (44).

(46) If $\emptyset \in B$, then $B=\{\emptyset\}$.

Proof: For every $a$ such that $a \in B$ holds $a=\emptyset$ by [4, (34)].

Let us consider $B$ and $n$. Note that $B \frown n$ is antichain-like.

Let us consider $T$. Let us observe that there exists a subset of $T^{*}$ which is non empty and antichain-like and $T^{\frown} n$ is non empty.

A Polish-language of $T$ is a non empty, antichain-like subset of $T^{*}$.

A Polish arity-function of $T$ is a function from $T$ into $\mathbb{N}$ and is defined by

(Def. 18) there exists $a$ such that $a \in T$ and $i t(a)=0$.

One can verify that every Polish-language of $T$ is non empty, antichain-like, and finite sequence-membered.

In the sequel $\alpha$ denotes a Polish arity-function of $T$ and $U, V, W$ denote Polish-languages of $T$.

Let us consider $T$ and $\alpha$. Let $t$ be an element of $T$. Let us observe that the functor $\alpha(t)$ yields a natural number. Let us consider $U$. Note that the Polishexpression layer $(T, \alpha, U)$ is defined by

(Def. 19) for every $a, a \in i t$ iff there exists an element $t$ of $T$ and there exists an element $u$ of $T^{*}$ such that $a=t^{\frown} u$ and $u \in U^{\frown} \alpha(t)$.

Let us consider $B$ and $p$. We say that $p$ is $B$-headed if and only if

(Def. 20) there exists $q$ and there exists $r$ such that $q \in B$ and $p=q^{\frown} r$.

Let us consider $P$. We say that $P$ is $B$-headed if and only if

(Def. 21) for every $p$ such that $p \in P$ holds $p$ is $B$-headed.

Now we state the propositions:

(47) If $p$ is $B$-headed and $B \subseteq C$, then $p$ is $C$-headed.

(48) If $P$ is $B$-headed and $B \subseteq C$, then $P$ is $C$-headed.

Let us consider $B$ and $P$. Observe that $B \frown P$ is $B$-headed.

Now we state the propositions:

(49) If $p$ is $(B \frown C)$-headed, then $p$ is $B$-headed.

(50) $B$ is $B$-headed. The theorem is a consequence of (3).

Let us consider $B$. Let us observe that there exists a finite sequence-membered set which is $B$-headed.

Let $P$ be a $B$-headed, finite sequence-membered set. Let us note that every subset of $P$ is $B$-headed.

Let us consider $S$. Let us observe that there exists a finite sequence-membered set which is non empty and $S$-headed. 
Now we state the proposition:

(51) $S \frown(m+n)$ is $(S \frown m)$-headed. The theorem is a consequence of (10).

Let us consider $S$ and $p$. The functor $S$-head $(p)$ yielding a finite sequence is defined by

(Def. 22) (i) it $\in S$ and there exists $r$ such that $p=i t^{\frown} r$, if $p$ is $S$-headed,

(ii) $i t=\emptyset$, otherwise.

The functor $S$-tail $(p)$ yielding a finite sequence is defined by

(Def. 23) $\quad p=(S \text {-head }(p))^{\frown} i t$.

Now we state the propositions:

(52) If $s \in S$, then $S$-head $(s \frown t)=s$ and $S$-tail $(s \frown t)=t$.

(53) If $s \in S$, then $S$-head $(s)=s$ and $S$-tail $(s)=\emptyset$. The theorem is a consequence of $(52)$.

Let us consider $S, T$, and $u$. Now we state the propositions:

(54) If $u \in S \frown T$, then $S$-head $(u) \in S$ and $S$-tail $(u) \in T$. The theorem is a consequence of (52).

(55) $\quad$ If $S \subseteq T$ and $u$ is $S$-headed, then $S$-head $(u)=T$-head $(u)$ and $S$-tail $(u)=$ $T$-tail $(u)$. The theorem is a consequence of (52).

Now we state the propositions:

(56) Suppose $s$ is $S$-headed. Then

(i) $s^{\frown} t$ is $S$-headed, and

(ii) $S$-head $(s \frown t)=S$-head $(s)$, and

(iii) $S$-tail $\left(s^{\curvearrowleft} t\right)=(S \text {-tail }(s))^{\frown} t$.

The theorem is a consequence of (52).

(57) If $m+1 \leqslant n$ and $s \in S \frown n$, then $s$ is $(S \frown m)$-headed and $S \frown m$-tail $(s)$ is $S$-headed. The theorem is a consequence of (51), (10), (54), and (7).

(58) (i) $s$ is $(S-0)$-headed, and

(ii) $S \frown 0-\operatorname{head}(s)=\emptyset$, and

(iii) $S \frown 0$-tail $(s)=s$.

The theorem is a consequence of (4) and (52).

Let us consider $T$ and $\alpha$. One can verify that the $\operatorname{Polish} \operatorname{atoms}(T, \alpha)$ is non empty and antichain-like.

Let us consider $U$. Let us observe that the Polish-expression layer $(T, \alpha, U)$ is non empty and antichain-like.

One can verify that the Polish-expression layer $(T, \alpha, U)$ yields a Polishlanguage of $T$. The Polish operations $(T, \alpha)$ yielding a subset of $T$ is defined by the term 
(Def. 24) $\{t$, where $t$ is an element of $T: \alpha(t) \neq 0\}$.

Let us consider $n$. Let us note that the Polish-expression hierarchy $(T, \alpha, n)$ is antichain-like and non empty.

One can check that the Polish-expression hierarchy $(T, \alpha, n)$ yields a Polishlanguage of $T$. The functor Polish-WFF-set $(T, \alpha)$ yielding a Polish-language of $T$ is defined by the term

(Def. 25) the Polish-expression $\operatorname{set}(T, \alpha)$.

A Polish WFF of $T$ and $\alpha$ is an element of Polish-WFF-set $(T, \alpha)$. Let $t$ be an element of $T$. The Polish operation $(T, \alpha, t)$ yielding a function from Polish-WFF-set $(T, \alpha) \frown \alpha(t)$ into Polish-WFF-set $(T, \alpha)$ is defined by the term

(Def. 26) the Polish operation $(T, \alpha, \alpha(t), t)$.

Assume $\alpha(t)=1$. The functor Polish-unOp $(T, \alpha, t)$ yielding a unary operation on Polish-WFF-set $(T, \alpha)$ is defined by the term

(Def. 27) the Polish operation $(T, \alpha, t)$.

Assume $\alpha(t)=2$. The functor Polish-binOp $(T, \alpha, t)$ yielding a binary operation on Polish-WFF-set $(T, \alpha)$ is defined by

(Def. 28) for every $u$ and $v$ such that $u, v \in$ Polish-WFF-set $(T, \alpha)$ holds $i t(u, v)=$ (the Polish operation $(T, \alpha, t))\left(u^{\curvearrowleft} v\right)$.

In the sequel $\varphi, \psi$ denote Polish WFFs of $T$ and $\alpha$.

Let us consider $X$ and $Y$. Let $F$ be a partial function from $X$ to $2^{Y}$. We say that $F$ is exhaustive if and only if

(Def. 29) for every $a$ such that $a \in Y$ there exists $b$ such that $b \in \operatorname{dom} F$ and $a \in F(b)$.

Let $X$ be a non empty set. Observe that there exists a finite sequence of elements of $2^{X}$ which is non exhaustive and disjoint valued.

Now we state the proposition:

(59) Let us consider a partial function $F$ from $X$ to $2^{Y}$. Then $F$ is not exhaustive if and only if there exists $a$ such that $a \in Y$ and for every $b$ such that $b \in \operatorname{dom} F$ holds $a \notin F(b)$.

Let us consider $T$. Let $B$ be a non exhaustive, disjoint valued finite sequence of elements of $2^{T}$. The Polish arity from list $B$ yielding a Polish arity-function of $T$ is defined by the term

(Def. 30) the arity from list $B$.

One can check that there exists an antichain-like, finite sequence-membered set which has non empty elements and there exists a Polish-language which is non trivial and every antichain-like, finite sequence-membered set which is non trivial has also non empty elements. 
Let us consider $S, n$, and $m$. Let $p$ be an element of $S \frown(n+1+m)$. The functor $\operatorname{decomp}(S, n, m, p)$ yielding an element of $S$ is defined by the term (Def. 31) $S$-head $(S \frown n$-tail $(p))$.

Let $p$ be an element of $S \frown n$. The functor $\operatorname{decomp}(S, n, p)$ yielding a finite sequence of elements of $S$ is defined by

(Def. 32) $\operatorname{dom} i t=\operatorname{Seg} n$ and for every $m$ such that $m \in \operatorname{Seg} n$ there exists $k$ such that $m=k+1$ and $i t(m)=S$-head $(S \frown k$-tail $(p))$.

Now we state the propositions:

(60) Let us consider an element $s$ of $S \frown n$, and an element $t$ of $T \frown n$. If $S \subseteq T$ and $s=t$, then $\operatorname{decomp}(S, n, s)=\operatorname{decomp}(T, n, t)$.

ProOF: $\operatorname{Set} p=\operatorname{decomp}(S, n, s)$. Set $q=\operatorname{decomp}(T, n, t)$. For every $a$ such that $a \in \operatorname{Seg} n$ holds $p(a)=q(a)$ by (17), [4, (1)], (57), (55).

(61) Let us consider an element $q$ of $S \frown 0$. Then $\operatorname{decomp}(S, 0, q)=\emptyset$.

(62) Let us consider an element $q$ of $S \frown n$. Then $\operatorname{len} \operatorname{decomp}(S, n, q)=n$.

(63) Let us consider an element $q$ of $S \frown 1$. Then $\operatorname{decomp}(S, 1, q)=\langle q\rangle$. The theorem is a consequence of (7), (58), (53), and (62).

(64) Let us consider elements $p, q$ of $S$, and an element $r$ of $S \frown 2$. Suppose $r=p \frown q$. Then $\operatorname{decomp}(S, 2, r)=\langle p, q\rangle$. The theorem is a consequence of (58), (52), (7), (53), and (62).

(65) Polish-WFF-set $(T, \alpha)$ is $T$-headed. The theorem is a consequence of (28), (23), and (21).

(66) The Polish-expression hierarchy $(T, \alpha, n)$ is $T$-headed. The theorem is a consequence of (26) and (65).

Let us consider $T$, $\alpha$, and $\varphi$. The functor Polish-WFF-head $\varphi$ yielding an element of $T$ is defined by the term

(Def. 33) $T$-head $(\varphi)$.

Let us consider $n$. Let $\varphi$ be an element of the Polish-expression hierarchy $(T$, $\alpha, n)$. The functor Polish-WFF-head $\varphi$ yielding an element of $T$ is defined by the term

(Def. 34) $T$-head $(\varphi)$.

Let us consider $\varphi$. The Polish arity $\varphi$ yielding a natural number is defined by the term

(Def. 35) $\quad \alpha($ Polish-WFF-head $\varphi)$.

Let us consider $n$. Let $\varphi$ be an element of the Polish-expression hierarchy $(T$, $\alpha, n)$. The Polish arity $\varphi$ yielding a natural number is defined by the term

(Def. 36) $\alpha($ Polish-WFF-head $\varphi)$.

Now we state the propositions: 
(67) $T$-tail $(\varphi) \in$ Polish-WFF-set $(T, \alpha) \frown($ the Polish arity $\varphi)$. The theorem is a consequence of (32) and (52).

(68) Let us consider an element $\varphi$ of the Polish-expression hierarchy $(T, \alpha$, $n+1)$. Then $T$-tail $(\varphi) \in($ the Polish-expression hierarchy $(T, \alpha, n)) \frown$ (the Polish arity $\varphi$ ). The theorem is a consequence of (23) and (52).

Let us consider $T, \alpha$, and $\varphi$. The functor $(T, \alpha)$-tail $\varphi$ yielding an element of Polish-WFF-set $(T, \alpha) \frown$ (the Polish arity $\varphi$ ) is defined by the term

(Def. 37) $T$-tail $(\varphi)$.

Now we state the proposition:

(69) If $T$-head $(\varphi) \in$ the $\operatorname{Polish}$ atoms $(T, \alpha)$, then $\varphi=T$-head $(\varphi)$. The theorem is a consequence of (67) and (6).

Let us consider $T, \alpha$, and $n$. Let $\varphi$ be an element of the Polish-expression hierarchy $(T, \alpha, n+1)$. The functor $(T, \alpha)$-tail $\varphi$ yielding an element of (the Polishexpression hierarchy $(T, \alpha, n))^{-}($the Polish arity $\varphi)$ is defined by the term

(Def. 38) $T$-tail $(\varphi)$.

Let us consider $\varphi$. The functor Polish-WFF-args $\varphi$ yielding a finite sequence of elements of Polish-WFF-set $(T, \alpha)$ is defined by the term

(Def. 39) decomp(Polish-WFF-set $(T, \alpha)$, the Polish arity $\varphi,(T, \alpha)$-tail $\varphi)$.

Let us consider $n$. Let $\varphi$ be an element of the Polish-expression hierarchy $(T$, $\alpha, n+1)$. The functor Polish-WFF-args $\varphi$ yielding a finite sequence of elements of the Polish-expression hierarchy $(T, \alpha, n)$ is defined by the term

(Def. 40) decomp(the Polish-expression hierarchy $(T, \alpha, n)$, the Polish arity $\varphi$, $(T, \alpha)$-tail $\varphi)$.

Now we state the propositions:

(70) Let us consider an element $t$ of $T$, and $u$.

Suppose $u \in$ Polish-WFF-set $(T, \alpha) \frown \alpha(t)$.

Then $T$-tail $(($ the Polish operation $(T, \alpha, t))(u))=u$. The theorem is a consequence of (52).

(71) Suppose $\varphi \in$ the Polish-expression hierarchy $(T, \alpha, n+1)$.

Then rng Polish-WFF-args $\varphi \subseteq$ the Polish-expression hierarchy $(T, \alpha, n)$. The theorem is a consequence of (60) and (26).

(72) Let us consider a finite sequence $p$, a function $f$ from $Y$ into $D$, and a function $g$ from $Z$ into $D$. Suppose $\operatorname{rng} p \subseteq Y$ and $\operatorname{rng} p \subseteq Z$ and for every $a$ such that $a \in \operatorname{rng} p$ holds $f(a)=g(a)$. Then $f \cdot p=g \cdot p$.

Proof: Reconsider $p_{1}=p$ as a finite sequence of elements of $Y$. Reconsider $q=f \cdot p_{1}$ as a finite sequence. Reconsider $p_{2}=p$ as a finite sequence of elements of $Z$. Reconsider $r=g \cdot p_{2}$ as a finite sequence. $q=r$ by [6, (33)], [4, (1)], [7, (13), (3)]. 
Let us consider $T, \alpha$, and $D$. The Polish recursion-domain $(\alpha, D)$ yielding a subset of $T \times D^{*}$ is defined by the term

(Def. 41) $\{\langle t, p\rangle$, where $t$ is an element of $T, p$ is a finite sequence of elements of $D: \operatorname{len} p=\alpha(t)\}$.

A Polish recursion-function of $\alpha$ and $D$ is a function from the Polish recursiondomain $(\alpha, D)$ into $D$. From now on $f$ denotes a Polish recursion-function of $\alpha$ and $D$ and $\gamma, \gamma_{1}, \gamma_{2}$ denote functions from Polish-WFF-set $(T, \alpha)$ into $D$.

Let us consider $T, \alpha, D, f$, and $\gamma$. We say that $\gamma$ is $f$-recursive if and only if

(Def. 42) for every $\varphi, \gamma(\varphi)=f(\langle T$-head $(\varphi), \gamma \cdot$ Polish-WFF-args $\varphi\rangle)$.

Now we state the proposition:

(73) If $\gamma_{1}$ is $f$-recursive and $\gamma_{2}$ is $f$-recursive, then $\gamma_{1}=\gamma_{2}$. The theorem is a consequence of (36), (17), (33), (52), (60), (72), and (37).

From now on $L$ denotes a non trivial Polish-language, $\beta$ denotes a Polish arity-function of $L, g$ denotes a Polish recursion-function of $\beta$ and $D, J, J_{1}$ denote subsets of Polish-WFF-set $(L, \beta), H$ denotes a function from $J$ into $D$, $H_{1}$ denotes a function from $J_{1}$ into $D$.

Let us consider $L, \beta, D, g, J$, and $H$. We say that $H$ is $g$-recursive if and only if

(Def. 43) for every Polish WFF $\varphi$ of $L$ and $\beta$ such that $\varphi \in J$ and rng Polish-WFF-args $\varphi \subseteq J$ holds $H(\varphi)=g(\langle L-\operatorname{head}(\varphi), H \cdot$ Polish-WFF-args $\varphi\rangle)$.

Now we state the propositions:

(74) There exists $J$ and there exists $H$ such that $J=$ the Polish-expression hierarchy $(L, \beta, n)$ and $H$ is $g$-recursive.

Proof: Define $\mathcal{X}$ [natural number] $\equiv$ there exists $J$ and there exists $H$ such that $J=$ the Polish-expression hierarchy $\left(L, \beta, \$_{1}\right)$ and $H$ is $g$-recursive. For every $n, \mathcal{X}[n]$ from [2, Sch. 2].

(75) There exists a function $\gamma$ from Polish-WFF-set $(L, \beta)$ into $D$ such that $\gamma$ is $g$-recursive.

Proof: Set $W=$ Polish-WFF-set $(L, \beta)$. Define $\mathcal{X}$ [object, object] $\equiv$ there exists $n$ and there exists $J_{1}$ and there exists $H_{1}$ such that $J_{1}=$ the Polishexpression hierarchy $(L, \beta, n)$ and $H_{1}$ is $g$-recursive and $\$_{1} \in J_{1}$ and $\$_{2}=$ $H_{1}\left(\$_{1}\right)$. For every $a$ such that $a \in W$ there exists $b$ such that $b \in D$ and $\mathcal{X}[a, b]$ by $(28),(74),[8,(5)]$. Consider $\gamma$ being a function from $W$ into $D$ such that for every $a$ such that $a \in W$ holds $\mathcal{X}[a, \gamma(a)]$ from $[8$, Sch. 1].

(76) Let us consider an element $t$ of $L$. Then the Polish operation $(L, \beta, t)$ is one-to-one. 
Proof: Set $f=$ the Polish operation $(L, \beta, t)$. For every $a$ and $b$ such that $a, b \in \operatorname{dom} f$ and $f(a)=f(b)$ holds $a=b$ by [4, (33)].

(77) Let us consider elements $t, u$ of $L$. Suppose $\operatorname{rng}($ the Polish operation $(L$, $\beta, t))$ meets $\operatorname{rng}($ the $\operatorname{Polish} \operatorname{operation}(L, \beta, u))$. Then $t=u$. The theorem is a consequence of (43).

(78) Let us consider an element $t$ of $L$, and $a$. Suppose $a \in \operatorname{dom}($ the Polish operation $(L, \beta, t))$. Then there exists $p$ such that

(i) $p=($ the Polish operation $(L, \beta, t))(a)$, and

(ii) $L$-head $(p)=t$.

The theorem is a consequence of (52).

Let us consider $L, \beta$, an element $t$ of $L$, and a Polish WFF $\varphi$ of $L$ and $\beta$. Now we state the proposition:

(79) Polish-WFF-head $\varphi=t$ if and only if there exists an element $u$ of $\operatorname{Polish}-W F F-\operatorname{set}(L, \beta) \frown \beta(t)$ such that $\varphi=($ the $\operatorname{Polish} \operatorname{operation}(L, \beta$, $t))(u)$. The theorem is a consequence of $(52)$.

Let us assume that $\beta(t)=1$. Now we state the propositions:

(80) If Polish-WFF-head $\varphi=t$, then there exists a Polish WFF $\psi$ of $L$ and $\beta$ such that $\varphi=(\operatorname{Polish}-u n O p(L, \beta, t))(\psi)$. The theorem is a consequence of (79) and (7).

(81) (i) Polish-WFF-head $((\operatorname{Polish}-\operatorname{unOp}(L, \beta, t))(\varphi))=t$, and

(ii) Polish-WFF-args $((\operatorname{Polish}-\operatorname{unOp}(L, \beta, t))(\varphi))=\langle\varphi\rangle$.

The theorem is a consequence of (7), (79), (70), and (63).

Now we state the proposition:

(82) Suppose $\beta(t)=2$. Then suppose Polish-WFF-head $\varphi=t$. Then there exist Polish WFFs $\psi, H$ of $L$ and $\beta$ such that $\varphi=(\operatorname{Polish}-\operatorname{binOp}(L, \beta, t))$ $(\psi, H)$. The theorem is a consequence of $(79),(6)$, and (7).

Now we state the propositions:

(83) Let us consider an element $t$ of $L$. Suppose $\beta(t)=2$. Let us consider Polish WFFs $\varphi, \psi$ of $L$ and $\beta$. Then

(i) Polish-WFF-head(Polish-binOp $(L, \beta, t))(\varphi, \psi)=t$, and

(ii) Polish-WFF-args $(\operatorname{Polish}-\operatorname{binOp}(L, \beta, t))(\varphi, \psi)=\langle\varphi, \psi\rangle$.

The theorem is a consequence of (7), (11), (79), (64), and (70).

(84) Let us consider a Polish WFF $\varphi$ of $L$ and $\beta$. Then $\varphi \in$ the Polish $\operatorname{atoms}(L, \beta)$ if and only if the Polish arity $\varphi=0$. The theorem is a consequence of (53), (67), and (6). 
(85) Let us consider a function $\gamma$ from Polish-WFF-set $(L, \beta)$ into $D$, an element $t$ of $L$, and a Polish WFF $\varphi$ of $L$ and $\beta$. Suppose $\gamma$ is $g$-recursive and $\beta(t)=1$. Then $\gamma((\operatorname{Polish}-\operatorname{unOp}(L, \beta, t))(\varphi))=g(t,\langle\gamma(\varphi)\rangle)$. The theorem is a consequence of (81).

Let us consider $S$. Let $p$ be a finite sequence of elements of $S$. The functor Flat $(p)$ yielding an element of $S \frown \operatorname{len} p$ is defined by

(Def. 44) $\operatorname{decomp}(S, \operatorname{len} p, i t)=p$.

Let us consider $L$ and $\beta$.

A substitution of $L$ and $\beta$ is a partial function from the $\operatorname{Polish} \operatorname{atoms}(L$, $\beta$ ) to Polish-WFF-set $(L, \beta)$. Let $s$ be a substitution of $L$ and $\beta$. The functor Subst $s$ yielding a Polish recursion-function of $\beta$ and $\operatorname{Polish}-W F F-\operatorname{set}(L, \beta)$ is defined by

(Def. 45) for every element $t$ of $L$ and for every finite sequence $p$ of elements of Polish-WFF-set $(L, \beta)$ such that len $p=\beta(t)$ holds if $t \in \operatorname{dom} s$, then $i t(t, p)=s(t)$ and if $t \notin \operatorname{dom} s$, then $i t(t, p)=t \frown \operatorname{Flat}(p)$.

Let $\varphi$ be a Polish WFF of $L$ and $\beta$. The functor $s[\varphi]$ yielding a Polish WFF of $L$ and $\beta$ is defined by

(Def. 46) there exists a function $H$ from Polish-WFF-set $(L, \beta)$ into Polish-WFF-set $(L, \beta)$ such that $H$ is (Subst $s)$-recursive and $i t=H(\varphi)$.

Now we state the proposition:

(86) Let us consider a substitution $s$ of $L$ and $\beta$, and a Polish WFF $\varphi$ of $L$ and $\beta$. If $s=\emptyset$, then $s[\varphi]=\varphi$.

Proof: Set $W=$ Polish-WFF-set $(L, \beta)$. Set $g=$ Subst $s$. Set $\gamma=\operatorname{id}_{W} \cdot \gamma$ is $g$-recursive by $(62),[6,(32),(33)],[7,(3),(17),(13)]$.

\section{REFERENCES}

[1] Grzegorz Bancerek. Cardinal numbers Formalized Mathematics, 1(2):377-382, 1990.

[2] Grzegorz Bancerek. The fundamental properties of natural numbers Formalized Mathematics, 1(1):41-46, 1990.

[3] Grzegorz Bancerek. The ordinal numbers. Formalized Mathematics, 1(1):91-96, 1990.

[4] Grzegorz Bancerek and Krzysztof Hryniewiecki. Segments of natural numbers and finite sequences. Formalized Mathematics, 1(1):107-114, 1990.

[5] Czesław Byliński. Binary operations. Formalized Mathematics, 1(1):175-180, 1990.

[6] Czesław Byliński. Finite sequences and tuples of elements of a non-empty sets. Formalized Mathematics, 1(3):529-536, 1990.

[7] Czesław Byliński. Functions and their basic properties Formalized Mathematics, 1(1): 55-65, 1990.

[8] Czesław Byliński. Functions from a set to a set Formalized Mathematics, 1(1):153-164, 1990.

[9] Czesław Byliński. Partial functions. Formalized Mathematics, 1(2):357-367, 1990.

[10] Czesław Byliński. Some basic properties of sets Formalized Mathematics, 1(1):47-53, 1990. 
[11] Agata Darmochwał. Finite sets. Formalized Mathematics, 1(1):165-167, 1990.

[12] Joanna Golińska-Pilarek and Taneli Huuskonen. Logic of descriptions. A new approach to the foundations of mathematics and science. Studies in Logic, Grammar and Rhetoric, 40(27), 2012.

[13] Joanna Golińska-Pilarek and Taneli Huuskonen. Grzegorczyk's non-Fregean logics. In Rafał Urbaniak and Gillman Payette, editors, Applications of Formal Philosophy: The Road Less Travelled, Logic, Reasoning and Argumentation. Springer, 2015.

[14] Andrzej Grzegorczyk. Filozofia logiki i formalna LOGIKA NIESYMPLIFIKACYJNA. Zagadnienia Naukoznawstwa, XLVII(4), 2012. In Polish.

[15] Jan Łukasiewicz. Uwagi o aksjomacie Nicoda i 'dedukcji uogólniającej'. In Ksiega pamiatkowa Polskiego Towarzystwa Filozoficznego, Lwów, 1931. In Polish.

[16] Andrzej Nędzusiak. Probability Formalized Mathematics, 1(4):745-749, 1990.

[17] Beata Padlewska. Families of sets Formalized Mathematics, 1(1):147-152, 1990.

[18] Zinaida Trybulec. Properties of subsets Formalized Mathematics, 1(1):67-71, 1990.

[19] Edmund Woronowicz. Relations and their basic properties. Formalized Mathematics, 1 (1):73-83, 1990.

Received April 30, 2015 